\title{
Cryptococcal meningitis and immune reconstitution inflammatory syndrome in a pediatric patient with HIV after switching to second line antiretroviral therapy: a case report
}

\author{
Ssegujja Boniface Joseph Otto ${ }^{1 *}$, Paul E. George ${ }^{2}$, Rebecca Mercedes ${ }^{2}$ and Nicolette Nabukeera-Barungi ${ }^{1}$
}

\begin{abstract}
Background: Cryptococcal meningitis (CCM) is a common and deadly disease among HIV-infected patients. Notable about CCM is its association with the immune reconstitution inflammatory syndrome (IRIS). Though it has been posited a switch from first to second-line antiretroviral therapy (ART) can induce CCM IRIS, a case presentation of CCM IRIS has not been published.

Case presentation: A 10-year-old, HIV-infected girl who initially presented with severe headache and new-onset seizures, with cerebrospinal fluid that returned antigen, India Ink, and culture positive for Cryptococcus neoformans. Notably, 8 weeks prior to seizures, she had switched from first line to second-line ART (abacavir-lamivudineefavirenz to zidovudine-lamivudine-lopinavir/ritonavir) due to virologic failure, with a viral load of 224,000 copies/ milliliter. At time of seizures and 8 weeks on second-line ART, her viral load had reduced to 262 copies/milliliter. Her hospital course was prolonged, as she had ongoing headaches and developed bilateral cranial nerve VI palsies despite clearance of Cryptococcus from cerebrospinal fluid on antifungal therapy and therapeutic lumbar punctures. However, symptoms stabilized, and she was discharged with oral fluconazole. Cranial nerve palsies resolved 10 weeks post discharge and she has remained disease free.
\end{abstract}

Conclusions: We describe a case of CCM IRIS in a 10-year-old HIV infected child after changing to second-line ART. This case provides evidence that screening for cryptococcal antigenaemia prior to switch from first-line to secondline ART could be an important measure to prevent cryptococcal disease.

Keywords: Cryptococcus neoformans, HIV, Immune reconstitution inflammatory syndrome (IRIS), Cryptococcal meningitis (CCM), Antiretroviral therapy

\section{Background}

Cryptococcus neoformans, an encapsulated fungal pathogen, is one of the commonest causes of meningitis worldwide [1]. As an opportunistic infection, it has a predilection for disease in the immunosuppressed, notably HIV-infected individuals and most prevalent in subSaharan Africa [2]. Despite advances in antiretroviral

\footnotetext{
* Correspondence: ottobonnie87@gmail.com

${ }^{1}$ Department of Paediatrics and Child Health, Makerere University College of Health Sciences, P.O. Box 7062, Kampala, Uganda

Full list of author information is available at the end of the article
}

therapy (ART) efficacy and delivery in sub-Saharan Africa, the burden of cryptococcal disease and specifically cryptococcal meningitis (CCM) has remained high. However, for unclear reasons, CCM is less common in children than adults, with an annual incidence of about 10-50 cases per 100,000 HIV-infected children when compared with 120 cases per 100,000 HIV-infected adults [3]. Case series of cryptococcal disease have shown the peak age to be $5-10$ years, though one large series also showed increased incidence in children $<1$

(c) The Author(s). 2020 Open Access This article is distributed under the terms of the Creative Commons Attribution 4.0 International License (http://creativecommons.org/licenses/by/4.0/), which permits unrestricted use, distribution, and reproduction in any medium, provided you give appropriate credit to the original author(s) and the source, provide a link to the Creative Commons license, and indicate if changes were made. The Creative Commons Public Domain Dedication waiver (http://creativecommons.org/publicdomain/zero/1.0/) applies to the data made available in this article, unless otherwise stated. 
year [3-7]. Mortality remains high in children, with reported case fatality rates ranging $20-40 \%[3,5]$.

Notable about CCM is its association with the immune reconstitution inflammatory syndrome (IRIS). IRIS describes a paradoxical worsening of clinical symptoms driven by increased inflammatory processes against an underlying infection in patients with a reversal of immune suppression [8]. Cryptococcal-associated IRIS is particularly common, with studies showing prevalence of $8-50 \%$ in HIV-infected patients starting ART, and dangerous, with reported mortalities of $27-83 \%$ in Africa [9]. For this reason, the 2018 World Health Organization Guidelines recommend screening for Cryptococcus infection in adults and adolescents prior to initiation or reinitiation of ART, and to defer treatment of ART for 46 weeks in patients with CCM [10]. Of note, screening for Cryptococcus infection is not recommended in children due to the low incidence of cryptococcal disease in this age group and the recommendations make no mention of screening when switching from first to second or third-line ART.

Given that IRIS occurs with rapid immune reversal, it has been posited that ART-associated Cryptococcus with IRIS could occur with a switch from first to second-line ART [9]. However, to our knowledge no case report of this so-called unmasking cryptococcal IRIS has been published. Here, we describe a case of CCM IRIS in a 10-year-old HIV infected child after changing to secondline ART.

\section{Case presentation}

A 10-year-old HIV-infected girl who presented to Mulago National Referral Hospital in Kampala, Uganda with a new-onset, generalized tonic-clonic seizure, which resolved with rectal diazepam given in the hospital. The seizure was preceded by a severe frontal headache and subjective fevers for 3 days. Otherwise, she did not have rash, vomiting, diarrhea, night sweats, or weight loss at presentation. There were no known contacts with tuberculosis. On initial exam, she was well appearing, with no abnormalities in vital signs or neurologic examination.

Cerebrospinal fluid (CSF) results showed WBC of $0-1$ per high powered field (hpf), red blood cells (RBC) 1-2/ hpf, protein $43 \mathrm{mg} / \mathrm{dL}$, glucose $2.5 \mathrm{mmol} / \mathrm{L}$ (normal 3.34.4). Rapid cryptococcal antigen in CSF and blood were positive. An acid-fast stain and Indian ink stain were positive $(++)$ for yeast cells. An opening pressure was not obtained due to lack of supplies. Two days later, the CSF culture returned positive (++) for Cryptococcus neoformans, and she was diagnosed with cryptococcal meningitis. Bacterial meningitis and HIV encephalopathy were the other considerations. CD4 count at the time of presentation was 445 cells/milliliter.
She was diagnosed with HIV at 6 years of age. Since starting ART, she reported poor adherence, leading to treatment failure and switch to second-line therapy $(\mathrm{ABC}-3 \mathrm{TC}-\mathrm{EFV} \rightarrow \mathrm{AZT}-3 \mathrm{TC}-\mathrm{LPV} / \mathrm{r}) 8$ weeks prior to the onset of her current symptoms; viral load at that time was 224,000 copies/milliliter. Her mother reported excellent adherence, with no missed doses, since starting second-line line ART. Her most recent CD4 count was from 3 years prior at 366 cells/milliliter. There were no recorded cryptococcal antigen tests from the previous year.

On admission, she was started on amphotericin B deoxycholate $(0.8 \mathrm{mg} / \mathrm{kg} /$ day $)$ and high dose oral fluconazole $(12 \mathrm{mg} / \mathrm{kg} /$ day $)$, as flucytosine is not readily available. Given ongoing neurologic symptoms (headache, vomiting), several therapeutic lumbar punctures were performed throughout admission (day 2 of hospitalization 8 $\mathrm{mL}$ was drained, day $6,10 \mathrm{~mL}$, day $10,15 \mathrm{~mL}$ and day 18 , $12 \mathrm{~mL}$ ) with transient improvement in symptoms. Despite this, on day 16 of hospitalization she developed bilateral cranial nerve VI palsy (see Fig. 1). CSF analysis showed a negative India ink stain and culture; CSF cell counts were not obtained due to a shortage of reagents. A CT head with/without contrast from day 17 showed mild parenchymal edema of left parietal lobe, otherwise normal. HIV viral load obtained at that time was 262 copies/milliliter. Given persistent symptoms, her induction therapy of amphotericin B and high dose fluconazole was increased from 14 to 21 days. She did not receive other adjuvant therapy such as glucocorticoids.

At discharge on day 21, she remained with bilateral cranial nerve VI palsy, but otherwise asymptomatic and seizure-free. Her cranial nerve defects resolved within 10 weeks of discharge and she has remained on oral fluconazole. Six months after discharge, she remains without seizures, cranial nerve palsies, or other sequala of CCM.

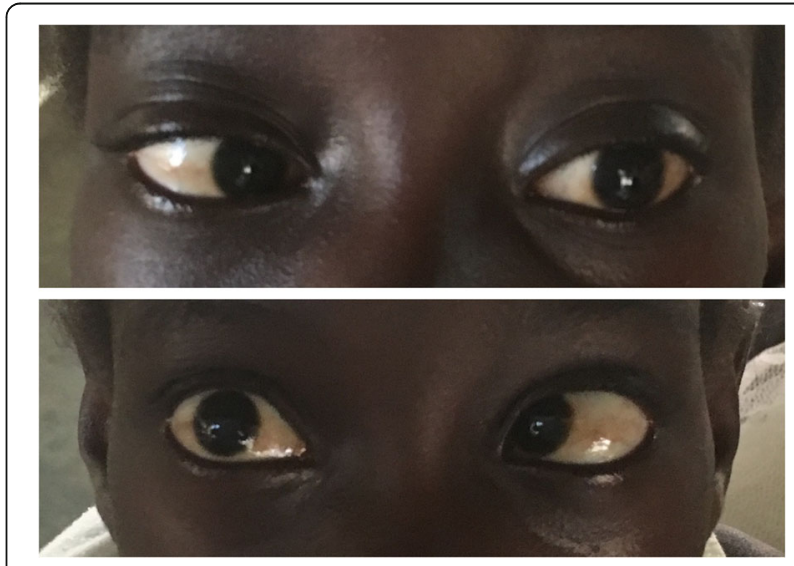

Fig. 1 Top Panel: Instructed to look left $\rightarrow$. Bottom Panel: Instructed to look right $\leftarrow$ 
This case report received IRB approval. We have obtained written consent from the primary caregiver of the child (the mother) and written assent from the child to publish the case report and associated images.

\section{Discussion}

This case highlights the occurrence of cryptococcal IRIS in the form of CCM in a pediatric, HIV-infected patient. This is the first case report of IRIS, a common sequalae of cryptococcal disease upon initiation of ART, described following switch from first to second-line ART.

In this case, the patient developed meningoencephalitic features including fever, headache, seizures and cranial nerve VI palsy. The most common symptoms seen in CCM are headache, fever, nausea, vomiting, dizziness, irritability and somnolence $[6,11]$. However, it may be virtually asymptomatic apart from a mild headache [6]. Unlike in adults, meningeal signs and an altered state of consciousness can be absent in HIV-positive children with CCM [12, 13]. For example, in a case report from South Africa involving seven pediatric patients with HIV and CCM, three patients presented with fever and without other localizing symptoms [14].

Cryptococcal IRIS presents as a clinical worsening or new presentation of cryptococcal disease after rapid reversal of immunodeficiency [15]. Cryptococcal IRIS is thought to be triggered by recovery of immune responses to cryptococcal species, resulting in exaggerated host inflammatory responses. In patients with HIV, the syndrome is driven by immune reconstitution due to ART. The syndrome has also been known to occur after solid organ transplantation (estimated incidence 4.8\%) [16] and in pregnancy [17]. Notably, in our patient, the CSF indices were not associated with an exaggerated inflammatory response. Previous studies have shown that HIV-infected children with CCM, unlike adults, are more likely to present with normal CSF indices, namely $\mathrm{WBC}<6$ cells/hpf [13]. Studies have also shown that the cytokine response in CCM IRIS is more robust in the peripheral blood than in the CSF [18].

However, per Haddow et al. [9], one clinical definition of an exaggerated inflammatory response is "meningitis ... with opening pressure $>20$ that is refractory to therapy." The increased opening pressure in cryptococcal meningitis is secondary to decreased reabsorption of CSF due to blockage by the cryptococcal capsule, indicating high burden of disease [19]. Presence of cryptococcal antigen has also been shown to inhibit leukocyte migration possibly accounting for the low WBC count despite relatively high CD4 count in this patient. The persistently present cryptococcus is what leads to the unregulated immune response as the $\mathrm{CD} 4$ recovers but may not particularly be reflected at the site of infection [20]. Although we were unable to obtain opening pressures, we presumed our patient remained with high intracranial pressures despite anti-fungal therapy given the persistent headaches, which were temporarily relieved with therapeutic lumbar punctures, and cranial nerve palsies she developed on day 16.

Two distinct modes of presentation of cryptococcal IRIS are recognized, paradoxical and ART-associated cryptococcal IRIS. Paradoxical cryptococcal IRIS presents as a worsening of disease or as a recurrent disease in the same or new anatomical sites, despite microbiological evidence of effective antifungal treatment. It occurs in up to one third of patients with cryptococcosis diagnosed before the initiation of ART [21, 22]. The patient in our case however had no evidence of ongoing cryptococcal disease prior to her 3 days of headaches, seizure, and subsequent diagnosis and treatment. A high index of suspicion is required for early diagnosis and treatment because cryptococcal meningitis IRIS sometimes does not present with overt clinical signs [13]. In our patient, her diagnosis was based on the proposed case definition and clinical checklist criteria from Haddow et al. [9] (Table 1).

The case is therefore more consistent with the later mode of presentation (ART-associated cryptococcal IRIS) in which new onset cryptococcosis occurs after ART is started in patients whom cryptococcosis was not recognized before treatment, occurring in up to $1 \%$ of patients [23]. Due to the difficulties in the differentiation between IRIS-associated disease (unmasking cryptococcal IRIS) and progression of untreated occult cryptococcosis in the context of persisting immunodeficiency, the term 'ART-associated cryptococcosis' has been preferred for both [9]. The diagnosis of IRIS in resourcelimited settings, our case included, has been made possible by adopting the clinical criteria checklist approach set by the International Network for the Study of HIV-

Table 1 Proposed case definitions for antiretroviral-therapyassociated cryptococcosis and unmasking cryptococcal immune reconstitution inflammatory syndrome

Antiretroviral therapy-associated cryptococcosis

1. Patient taking antiretroviral therapy (ART)

2. No recognized cryptococcal disease at ART initiation

3. Clinical disease worsening caused by cryptococcosis occurs after

initiation, re-introduction, or regimen switch after previous failure

4. Cryptococcal infection characterized by meningitis, CNS

complications, skin or soft-tissue lesions, lymphadenopathy, lung disease, or disseminated disease

Unmasking cryptococcal IRIS

1. Criteria for ART-associated cryptococcosis are met

2. Unusual, exaggerated, or heightened inflammatory manifestations

3. Event occurs early after ART initiation (typically, within 3 months)

4. Failure of ART excluded if possible (eg, $\geq 1.0 \log 10 \mathrm{copies} / \mathrm{mL}$ decrease in HIV-1 viral load by 8 weeks treatment)

Above proposed definitions taken from Haddow et al. Cryptococcal immune reconstitution inflammatory syndrome in HIV-1-infected individuals: proposed clinical case definitions. Lancet Infect Dis 2010;10(11):791-802 
associated IRIS since there is no definitive diagnostic test.

The onset of our patient's seizures was rapid, occurring just 3 days from the time of first complaint of fever and headache. This is consistent with ART-associated CCM which is often characterized by rapid development of severe illness, developing over a few days from the onset of symptoms [24], compared with the 1-2 week subacute course typically seen with CCM in patients not receiving ART [21]. From individually reported times of symptom onset, the median time after the start of ART was 9 weeks (IQR 2-26 weeks) in patients with paradoxical cryptococcal IRIS and 4 weeks (IQR 2-10 weeks) in those with ART-associated cryptococcosis [9]. The patient in this case report developed symptoms of CCM 8 weeks after switching from first to second-line ART.

Interestingly, our patient's CD4 of 445 cells/milliliter was higher than typically recorded for patients with IRIS. However, this CD4 count was taken at time of diagnosis of CCM, 8 weeks after initiation of second-line ART. As such, her CD4 count was likely significantly lower when starting second-line, as previous studies have shown rises of CD4 counts of 50-150 cells/milliliter within the first 6-12 weeks after starting protease-inhibitor secondline ART [25]. Further, IRIS is most likely to occur in the setting of a rapidly rising CD4 count [26], and weeks 6-18 have been shown to be weeks when CD4 counts rise the fastest [27]. Thus, while her CD4 count at diagnosis of CCM was unusually high, her overall clinical trajectory is typical for CCM IRIS.

ART-associated cryptococcosis is caused by either restoration of a Cryptococcus specific immune response (i.e., unmasking cryptococcal IRIS) or by persistent immunodeficiency while receiving ART [9]. Although the patient in this case report had evidence of ART failure with persistence of immunodeficiency prior to switching ART, there was no evidence of CCM. This case therefore correlates more with unmasking ART-associated cryptococcal IRIS, likely in the setting of immune restoration that occurred following the switch of ART, as evidenced by the drop in the viral load from 224,000 copies/ milliliter to 262 copies/ milliliter within a period of 3 months. While not required to make a diagnosis, a specific threshold for a reduction in viral load of greater than 1.0Log10 copies/ milliliter at the time of clinical event supports the diagnosis of IRIS [28]. In the case of our patient, the drop in the viral load following switch of ART was $2.9 \log 10$, significantly higher than 1.0 Log10 threshold. The significant drop in viral load allows for immune reconstitution to occur and therefore is used a surrogate marker of immune reconstitution. The best time to do viral load assays or the best threshold value or values have however not been experimentally validated [9]. The clinical distinction of the two causes of ART-associated cryptococcal IRIS is important because clinical management could be affected by this distinction and also because both causes are important in regions of high cryptococcal prevalence, our setting inclusive [9].

Subclinical antigenaemia is the overwhelming risk factor for ART-associated cryptococcosis, with the incidence nearly $33 \%$ in individuals with subclinical cryptococcal antigenaemia without pre-emptive therapy with fluconazole [29]. In our patient, it is possible that she had subclinical antigenaemia; however, no screening test was done prior to switching of ART. In addition, the World Health Organization guideline does not mention screening for children, only for adults and adolescents. The guideline recommends screening for cryptococcal antigen followed by pre-emptive antifungal therapy among cryptococcal antigen-positive people to prevent the development of invasive cryptococcal disease for adults and adolescents living with HIV who have a CD4 cell count $<100$ cells $/ \mathrm{mm}^{3}$ [10]. Furthermore, both the WHO and Ugandan national guidelines only recommends screening in patients prior to initiation or reinitiation of ART; it makes no mention of screening prior to switch from first to second or second to third line therapy. As such, our patient did not undergo cryptococcal screening prior to switch from first to second line therapy. Given that IRIS occurs with rapid immune reversal, it has been posited that ART-associated Cryptococcus with IRIS could occur with a switch from first to second-line ART [9]. However, to our knowledge no case of this so-called unmasking cryptococcal IRIS following switch from first to second-line therapy has been reported, either in adult or pediatric literature.

In conclusion, cryptococcal IRIS is thought to be triggered by recovery of immune responses to cryptococcal species, resulting in exaggerated host inflammatory responses. In patients with HIV, the syndrome is due to the immune reconstitution driven by effective ART, which could plausibly occur with a switch from first to second line ART. This case therefore highlights the need for future studies on cryptococcal antigenaemia in children not only at initiation of ART but also prior to switching between lines of therapy in children, adolescents, and adults, as such screening could have important clinical implications for the prevention of cryptococcal disease.

\section{Abbreviations}

ART: Antiretroviral therapy; CCM: Cryptococcal meningitis; CSF: Cerebrospinal fluid; IRIS: Immune reconstitution inflammatory syndrome

\section{Acknowledgements}

We would like the thank the patient and her caretaker for consenting to publication of her story.

Authors' contributions

SBJO wrote the initial draft of the manuscript. PEG conceptualized the case report and edited the final draft. RM edited the first and final draft. NBM oversaw the project. All authors read and approved the final manuscript. 


\section{Funding}

The authors have no funding to declare.

\section{Availability of data and materials}

Not applicable.

\section{Ethics approval and consent to participate}

This case report received IRB approval from Mulago Hospital Ethics and Research Committee. We have obtained written consent from the primary caregiver of the child (the mother) and written assent from the child to publish the case report and associated images. These documents are available upon request.

\section{Consent for publication}

We have obtained written consent from the primary caregiver of the child (the mother) and written assent from the child to publish the case report and associated images. These documents are available upon request.

\section{Competing interests}

The authors declare that they have no competing interests.

\section{Author details}

${ }^{1}$ Department of Paediatrics and Child Health, Makerere University College of Health Sciences, P.O. Box 7062, Kampala, Uganda. ${ }^{2}$ Baylor College of Medicine and Texas Children's Hospital, Houston, TX, USA.

Received: 27 July 2019 Accepted: 15 January 2020

Published online: 21 January 2020

\section{References}

1. Rajasingham R, Smith RM, Park BJ, Jarvis JN, Govender NP, Chiller TM, et al Global burden of disease of HIV-associated cryptococcal meningitis: an updated analysis. Lancet Infect Dis. 2017;17:873-81.

2. Kao C, Goldman DL. Cryptococcal disease in HIV-infected children. Curr Infect Dis Rep. 2016;18:27.

3. Meiring ST, Quan VC, Cohen C, Dawood H, Karstaedt AS, McCarthy KM, et al. A comparison of cases of paediatric-onset and adult-onset cryptococcosis detected through population-based surveillance, 2005-2007. Aids. 2012;26: 2307-14.

4. Joshi NS, Fisher BT, Prasad PA, Zaoutis TE. Epidemiology of cryptococcal infection in hospitalized children. Pediatr Infect Dis J. 2010;29:e91-5.

5. Luo FL, Tao YH, Wang YM, Li H. Clinical study of 23 pediatric patients with cryptococcosis. Eur Rev Med Pharmacol Sci. 2015;19:3801-10.

6. Severo CB, Xavier MO, Gazzoni AF, Severo LC. Cryptococcosis in children Paediatr Respir Rev. 2009;10:166-71.

7. Lizarazo J, Escandón P, Agudelo Cl, Castañeda E. Cryptococcosis in Colombian children and literature review. Mem Inst Oswaldo Cruz. 2014;109: 797-804.

8. Müller M, Wandel S, Colebunders R, Attia S, Furrer H, Egger M. Immune reconstitution inflammatory syndrome in patients starting antiretroviral therapy for HIV infection: a systematic review and meta-analysis. Lancet Infect Dis. 2010;10:251-61.

9. Haddow LJ, Colebunders R, Meintjes G, Lawn SD, Elliott JH, Manabe YC, et al. Cryptococcal immune reconstitution infl ammatory syndrome in HIV1 -infected individuals : proposed clinical case defi nitions. Lancet Infect Dis. 2010:10:791-802

10. WHO | Guidelines for the diagnosis, prevention and management of cryptococcal disease in HIV-infected adults, adolescents and children. World Health Organization; 2018. http://www.who.int/hiv/pub/guidelines/ cryptococcal-disease/en/. Accessed 23 Oct 2018.

11. Nyazika TK, Masanganise F, Hagen F, Bwakura-Dangarembizi MF, Ticklay IM, Robertson VJ. Cryptococcal meningitis presenting as a complication in HIVinfected children: a case series from sub-Saharan Africa. Pediatr Infect Dis J. 2016:35:979

12. Leggiadro RJ, Kline MW, Hughes WT. Extrapulmonary cryptococcosis in children with acquired immunodeficiency syndrome. Pediatr Infect Dis J. 1991;10:658-62.

13. Gumbo T, Kadzirange G, Mielke J, Gangaidzo IT, Hakim JG. Cryptococcus neoformans meningoencephalitis in African children with acquired immunodeficiency syndrome. Pediatr Infect Dis J. 2002;21:54-6.
14. Hassan H, Cotton MF, Rabie H. Complicated and protracted Cryptococcal disease in HIV-infected children. Pediatr Infect Dis J. 2015:34:62.

15. Singh N, Perfect JR. Immune reconstitution syndrome associated with opportunistic mycoses. Lancet Infect Dis. 2007;7:395-401.

16. Singh N, Lortholary $\mathrm{O}$, Alexander BD, Gupta KL, John GT, Pursell K, et al. An Immune Reconstitution Syndrome - Like Illness Associated with Cryptococcus neoformans Infection in Organ Transplant Recipients. 2005; 15240.

17. Singh N, Perfect JR. Immune reconstitution syndrome and exacerbation of infections after pregnancy. Clin Infect Dis. 2007:45:1192-9.

18. Wiesner DL, Boulware DR. Cryptococcus-related immune reconstitution inflammatory syndrome (IRIS): pathogenesis and its clinical implications Curr Fungal Infect Rep. 2011;5:252.

19. Abassi M, Boulware DR, Rhein J. Cryptococcal meningitis: diagnosis and management update. Curr Trop Med Rep. 2015:2:90-9.

20. Meya DB, Okurut S, Zziwa G, Rolfes MA, Kelsey M, Cose S, et al. Cellular immune activation in cerebrospinal fluid from Ugandans with Cryptococcal meningitis and immune reconstitution inflammatory syndrome. J Infect Dis. 2015;211:1597-606.

21. Kambugu A, Meya DB, Rhein J, O'Brien M, Janoff EN, Ronald AR, et al. Outcomes of cryptococcal meningitis in Uganda before and after the availability of highly active antiretroviral therapy. Clin Infect Dis. 2008;46: 1694-701.

22. Shelburne SA III, Darcourt J, White AC Jr, Greenberg SB, Hamill RJ, Atmar RL et al. The role of immune reconstitution inflammatory syndrome in AIDSrelated Cryptococcus neoformans disease in the era of highly active antiretroviral therapy. Clin Infect Dis. 2005;40:1049-52.

23. Murdoch DM, Venter WDF, Feldman C, Van Rie A. Incidence and risk factors for the immune reconstitution inflammatory syndrome in HIV patients in South Africa: a prospective study. AIDS (London, England). 2008;22:601-10

24. Woods ML, MacGinley R, Eisen DP, Allworth AM. HIV combination therapy: partial immune restitution unmasking latent cryptococcal infection. AIDS (London, England). 1998:12:1491-4

25. Deeks SG, Hecht FM, Swanson M, Elbeik T, Loftus R, Cohen PT, et al. HIV RNA and CD4 cell count response to protease inhibitor therapy in an urban AIDS clinic: response to both initial and salvage therapy. AIDS. 1999;13:F35.

26. Boulware DR, Callens S, Pahwa S. Pediatric HIV immune reconstitution inflammatory syndrome (IRIS). Curr Opin HIV AIDS. 2008;3:461-7.

27. Lewis J, Walker AS, Castro H, De Rossi A, Gibb DM, Giaquinto C, et al. Age and CD4 count at initiation of antiretroviral therapy in HIV-infected children: effects on long-term T-cell reconstitution. J Infect Dis. 2012;205:548-56.

28. Robertson J, Meier M, Wall J, Ying J, Fichtenbaum CJ. Immune reconstitution syndrome in HIV: validating a case definition and identifying clinical predictors in persons initiating antiretroviral therapy. Clin Infect Dis. 2006:42:1639-46.

29. Jarvis JN, Lawn SD, Vogt M, Bangani N, Wood R, Harrison TS. Screening for cryptococcal antigenemia in patients accessing an antiretroviral treatment program in South Africa. Clin Infect Dis. 2009:48:856-62.

\section{Publisher's Note}

Springer Nature remains neutral with regard to jurisdictional claims in published maps and institutional affiliations.

Ready to submit your research? Choose BMC and benefit from:

- fast, convenient online submission

- thorough peer review by experienced researchers in your field

- rapid publication on acceptance

- support for research data, including large and complex data types

- gold Open Access which fosters wider collaboration and increased citations

- maximum visibility for your research: over $100 \mathrm{M}$ website views per year

At BMC, research is always in progress.

Learn more biomedcentral.com/submissions 